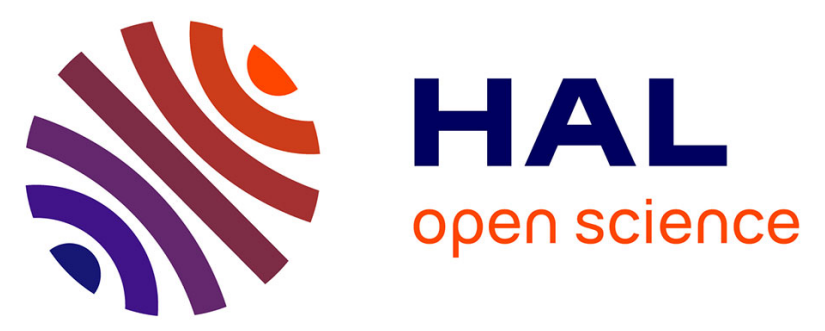

\title{
A Pragmatic Approach to Electronically Rewritable 'RF-Barcodes' Based on Non-Volatile CBRAM/MIM Switching Technology
}

\author{
M.P. Jayakrishnan, Arnaud Vena, Brice Sorli, Etienne Perret
}

\section{To cite this version:}

M.P. Jayakrishnan, Arnaud Vena, Brice Sorli, Etienne Perret. A Pragmatic Approach to Electronically Rewritable 'RF-Barcodes' Based on Non-Volatile CBRAM/MIM Switching Technology. 2020 XXXIIIrd General Assembly and Scientific Symposium of the International Union of Radio Science (URSI GASS), Aug 2020, Rome, Italy. 10.23919/URSIGASS49373.2020.9232256 . hal-03217713

\author{
HAL Id: hal-03217713 \\ https://hal.science/hal-03217713
}

Submitted on 5 May 2021

HAL is a multi-disciplinary open access archive for the deposit and dissemination of scientific research documents, whether they are published or not. The documents may come from teaching and research institutions in France or abroad, or from public or private research centers.
L'archive ouverte pluridisciplinaire $\mathbf{H A L}$, est destinée au dépôt et à la diffusion de documents scientifiques de niveau recherche, publiés ou non, émanant des établissements d'enseignement et de recherche français ou étrangers, des laboratoires publics ou privés. 


\title{
A Pragmatic Approach to Electronically Rewritable 'RF-Barcodes' Based on Non-Volatile CBRAM/MIM Switching Technology
}

\author{
Jayakrishnan M.P.*(1), Arnaud Vena ${ }^{(2)}$, Brice Sorli ${ }^{(2)}$, and Etienne Perret ${ }^{(1,3)}$ \\ (1) Université Grenoble Alpes, Grenoble INP, LCIS, 26000 Valence, France. \\ Email: jayakrishnan.mp@lcis.grenoble-inp.fr, etienne.perret@lcis.grenoble-inp.fr \\ (2) Institut d'Electronique et des Systèmes (IES), Université de Montpellier / CNRS, 34095 Montpellier, France. \\ (3) Institut Universitaire de France, 75005 Paris, France. \\ Email: arnaud.vena@umontpellier.fr,brice.sorli@ies.univ-montp2.fr
}

\begin{abstract}
In this article we present the concept of a practical approach to realization of a fully passive electronically rewritable chipless RFID tag. Integrated non-volatile and solid state Conductive Bridging Random Access Memory (CBRAM) based Metal-Insulator-Metal (MIM) switches are used to realize the rewritable functionality. A solution to the primary challenge of requirement of obtaining very low ON states resistances $(\sim<10 \Omega)$ in case of an RF switch, for efficient operating conditions is addressed in this paper. Here we propose a reconfigurable resonator design with a ground plane, which could operate efficiently for higher ON state resistances $(\sim>100 \Omega)$ of a CBRAM/MIM RF switch. A technique of data encoding in electronically rewritable dipole resonators, by effectively modulating the impedance states of integrated CBRAM/MIM switches is presented herewith in detail. This example is presented through a model design of a 3bit rewritable chipless RFID tag.
\end{abstract}

\section{Introduction}

Chipless RFID is an innovative breakthrough among RFID technology, in the recent years [1]. These identifiers are often called 'RF-Barcodes' due to their resemblance to optical barcode systems [2], [3]. In fact chipless RFID technology offers much more than an optical barcode identifier, in terms of functionalities like non-optical-lineof-sight readability, sensor integration capability, rewriteablity, and so on [4]-[7].

One of a perceptible question that impedes extensive commercial use of chipless RFID is cost and simplicity in comparison to an optical barcode system. An answer to this encumbering issue, even though in its budding stage, is the electronic rewriteablity offered to chipless RFID using CBRAM (Conductive Bridging Random Access Memory) switches [7], [8]. CBRAM or MIM (Metal Insulator Metal) switches are a group of non-volatile memory inspired switches, suitable for RF applications, derived from the well-known memristor concept [9]-[12]. A CBRAM/MIM switch is a simple electrochemical switch, in which two electrochemically asymmetric electrode pairs sandwich a dielectric compound, which is an ion-conductor and an electronic insulator (like chalcogenide glass, inomeric polymers etc.) [9], [11], [13]. Here the electrochemical asymmetry of electrodes means that one of the electrodes is an electrochemically active ion-donor metal (like silver, copper and similar) and the other is an inert ion-acceptor metal (gold, aluminum, tungsten and similar). Switching action is achieved by controlled formation (ON) and dissolution (OFF) of a conductive filament between the electrodes, through this ion-conductor layer. CBRAM switches are operated using low power DC pulses to change their impedance states.

The primary and major difference that distinguishes these switches from classic RF switches like semiconductor switches or MEMS technology is that CBRAM switches are essentially non-volatile, which means they do not require any power supply to maintain their state. This peculiar property makes these switches greatly reliable for passive and low power RF-systems. With ion-conductors like Nafion that are potentially printable, a scientific breakthrough enabling the out of 'clean room' fabrication of electronically reconfigurable $\mathrm{RF}$ and Microwave devices is envisaged [14]. Recently, we have shown (for the first time) that CBRAM switches could be integrated into a chipless RFID tag to enable electronically rewritable 'RF - Barcodes' or chipless RFID tags with comparable or even more technologically superior functionality to optical barcode systems [7], [8]. These chipless RFID tags are fabricated both on rigid as well as flexible substrates like office paper. However, as it would be the greatest challenge for any budding technologies, it is relevant for this optimized CBRAM technology as well, to prove its metal for a practical or out of laboratory environment.

In this article we try to present a pragmatic approach to design of electronically rewritable chipless RFID tags. These tags are optimized to operate at practical impedance states of integrated CBRAM RF-switches, fabricated using simple processes, in ambient environments [14] without involving any 'clean room' technologies.

\section{The Targeted Challenge}

CBRAM/MIM switch cells composed of Aluminum/Nafion/Copper or Aluminum/Nafion/Silver layer structures are utilized in all our studies [15]. These cells are operated using optimized DC pulses as reported in [14]. A critical requirement for RF application of these switches is that their low resistance state (LRS) or set state (or ON state) impedance should be of the order of a 
few ohms $(\sim<10 \Omega)$ and their high resistance states (HRS) or reset state (or OFF state) impedance should be essentially very high $(\sim>10 \mathrm{k} \Omega)$, to ensure a good degree of RF transmission and isolation respectively. A group of typical CBRAM cells with Aluminum/Nafion/Copper layer structure, used in our studies [15], are given in Fig. 1. These cells show an excellent operation with impedance states mostly of the order of $<10 \Omega$ and $>1$ $\mathrm{M} \Omega$ respectively for LRS and HRS. However, as it is a budding technology, without any in-depth optimizations, in terms of microfabrication clubbed together with material sciences. Sometimes due to some reasons that are not yet elucidated, or uncertain, like any imperfections in the fabrication process, possible contaminations of utilized materials, or with the intrusion of ambient environment, we could see some short comings in terms of the desired impedance levels.

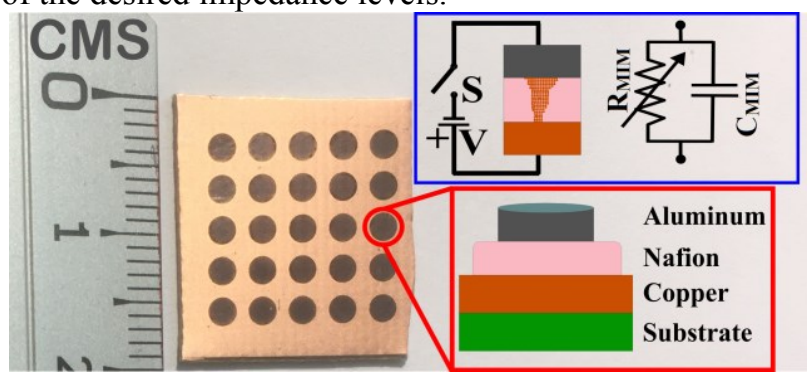

Fig. 1. Aluminum/Nafion/Copper based CBRAM/MIM cells, with zoom image showing cross section of layer architecture. Inset of the figure in blue border shows the way of application of DC voltage pulses for set (ON) process (reset or OFF process uses a reverse polarity), and the equivalent electrical model.

Practical ON/OFF or LRS/HRS cycles of a typical CBRAM cell from the group of switches in Fig. 1, are shown in Fig. 2. Here, one could see that the HRS levels are more or less stable at around $1 \mathrm{M} \Omega$. On the other hand, we could see that the LRS values are more spread in terms of impedance in the interval of $1 \Omega$ to few hundreds of ohms.

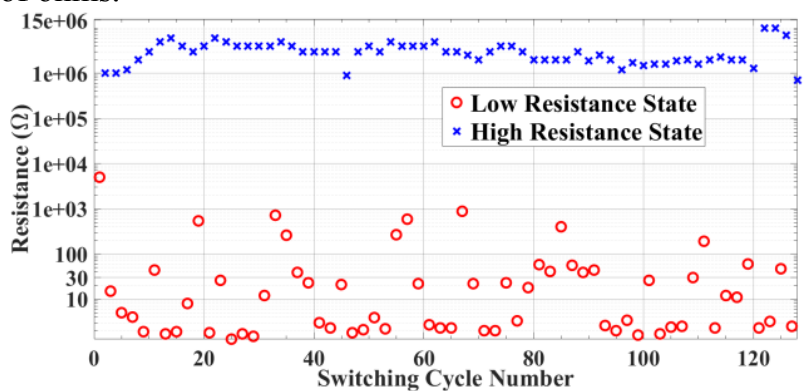

Fig. 2. 125 regular set/reset (ON/OFF) switching cycles of a typical CBRAM/MIM switch cell as shown in Fig. 1.

This phenomenon would introduce a possible, but undesired disparity to pre-coded states of a chipless RFID tag (as in [7]), in LRS. To overcome this challenge, in this article we propose and present a new concept of chipless RFID tag based on electronically rewritable resonators with integrated CBRAM switches. These resonators are resistant to impedance fluctuations at LRS, by limiting their requirement of impedance to $\sim 100 \Omega$ or more in respective LRSs.

\section{Proposed Design of an Electronically Rewritable Dipole-Barcode}

Proposed topology of rewritable resonator for chipless RFID tag is given in Fig. 3. This design is basically a shorted dipole with a ground plane. A CBRAM switch is integrated into this dipole at its center by modifying the trace width, to achieve a low OFF state capacitance. Construction and layer structure of the integrated CBRAM switch is shown as a zoom image in Fig. 3.

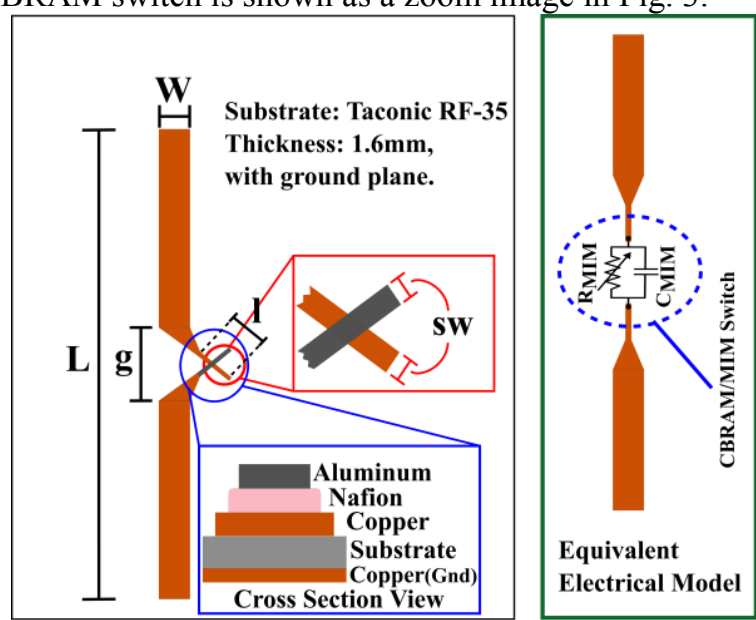

Fig. 3. Proposed electronically rewritable resonator with integrated CBRAM/MIM switch, for chipless RFID applications. Inset in green border shows equivalent circuit of dipole with integrated CBRAM/MIM switch. (L $=40 \mathrm{~mm}, \mathrm{~W}=3 \mathrm{~mm}, \mathrm{~g}=6 \mathrm{~mm}, \mathrm{l}=3 \mathrm{~mm}, \mathrm{sw}=200 \mu \mathrm{m})$

This structure is initially simulated using CST Microwave studio, and the observed RCS resonance response is given in Fig. 4. An electromagnetic plane wave polarized along length $L$ (see Fig. 4) of the dipole is used as excitation and an RCS probe placed in far-field of the structure is used to capture the backscatter response.

For fast simulations and smaller number of mesh cells, a CBRAM cell is approximated using a lumped $\mathrm{RC}$ parallel network with values calculated using the geometry of chosen design, and with knowledge from previous realizations [15]. Here in Fig. 4, one could observe that a weak resonances dip at $1.896 \mathrm{GHz}$ is seen for LRS around $\sim 1 \Omega$ and a very strong resonance dip at $2.704 \mathrm{GHz}$ for HRS around $1 \mathrm{M} \Omega$. However these resonance dips are suppressed and a flat RCS response is observed for LRS states around $\sim 100 \Omega$.

The working mechanism of this tag is simple and could be explained as follows. A CBRAM switch as presented here could be approximated as an RC parallel network (as shown in Fig. 1) whose resistance $\left(R_{M I M}\right)$ is similar to the measured switch impedance (filament resistance) and the capacitance $\left(C_{M I M}\right)$ is similar to calculated parallel plate capacitance of the switch due to its geometry and dielectric properties of ion conductor used [7]. Thus a circuit approximation for the presented network would be approximated like adding a RC parallel network as shown in Fig. 3, to the dipole. To further understand, one should recall that the total RCS response observed from a multiresonator based chipless RFID tag is an interference pattern as a result of a destructive or constructive 
interference of time cleaved scattering of electromagnetic energy, of two principle modes. First called the structural mode, which is an immediate reflection of energy from the tag (similar to optical reflection), and second the time delayed resonant response of each resonator present on the tag, called antenna mode [7], [16]. Here initially when the CBRAM switch is in LRS of the order of $1 \Omega\left(R_{M I M}=\right.$ $1 \Omega$ ), the capacitor $C_{M I M}$ of CBRAM is bypassed, hence forcing the dipole to resonate at a half wavelength mode. The presence of ground plane leads to excitement of cavity modes leading to a destructive interference among the structural and antenna modes of the tag, resulting in a dip at the resonance frequency of $1.896 \mathrm{GHz}$. However, one could observe that this resonant mode is not very strong and produces a dip only of the order of $\sim 5 \mathrm{~dB}$ with respect to the average RCS value over the bandwidth. Next, when the CBRAM switch is in HRS of the order of $1 \mathrm{M} \Omega$, the resistance of its equivalent circuit $\left(R_{M I M}=\right.$ $1 M \Omega$ ) is similar to an open circuit and thus adds the capacitance $C_{M I M}$ to the dipole, thus effectively reducing its effective electrical length and forcing it to resonate at a higher frequency of $2.704 \mathrm{GHz}$, at a higher order mode. This leads to excitation of strong cavity modes between the ground plane and resonator, resulting in a sharp resonant dip of the order of $\sim 15$ to $20 \mathrm{~dB}$ in the RCS response. Now if we look at the third case, when the CBRAM switch is in LRS but around $\sim 100 \Omega\left(\mathrm{R}_{\mathrm{MIM}}=\right.$ $100 \Omega$, we see no resonances at either of these frequencies. This is an effect of addition of such a high resistance to the total resonance structure, which greatly reduces the quality factor of the antenna mode response leading to weak interference with the structural mode and thus exhibits no notable resonant response. These inferences are drawn based on observation of electric and magnetic fields, and surface current patterns on the simulated model. These details are however, omitted from this article due to space constraints.

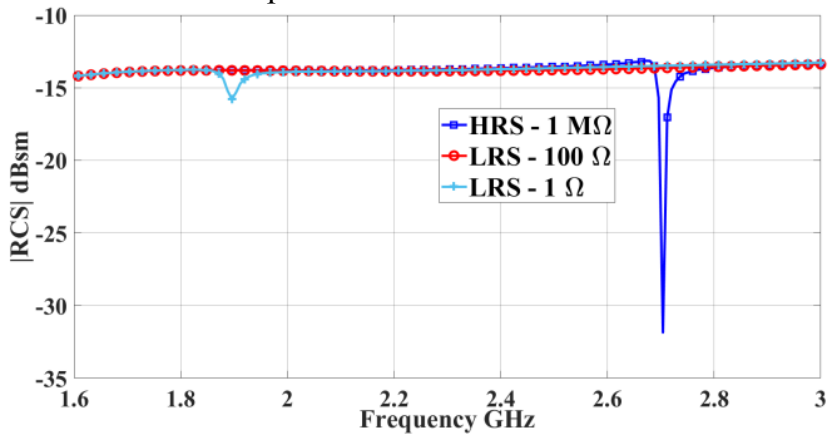

Fig. 4. Backscattered RCS response of electronically rewritable resonator presented in Fig. 3 for different impedance states of integrated CBRAM/MIM switch (full wave simulation).

Here as explained earlier, for practical considerations, we target on a solution to operate a resonator for rewritable chipless RFID applications, using an integrated RF switch with an LRS of the order of $100 \Omega$ and above in ON state. In this context, the obtained result is an affirmation that we could operate a resonator for chipless RFID in this resistance range (i.e. LRS $\sim 100 \Omega$ and $\mathrm{HRS} \sim 1 \mathrm{M} \Omega$ ), of CBRAM/MIM switches. Such a concept is quite unusual in most cases, where researchers target on the case of an RF switch with a very low LRS [16] in the ON state. The latter case however demands a quintessentially ideal behavior of an RF switch (i.e. LRS $\sim 1 \Omega$ ), which is very difficult to achieve in practical cases, especially using low cost fabrication techniques, as the one desired in this approach.

\section{Chipless RFID Tag based on Rewritable Dipole Barcodes}

In this section we present the proposed chipless RFID tag with three rewritable dipole resonators as in Fig. 5, with different lengths. Each dipole resonator could represent two states depending on the impedance state of integrated CBRAM switch (in the range LRS $\sim 100 \Omega$ and HRS $\sim 1$ $M \Omega)$. Thus we have a total of 8 distinct states as represented in Table 1, for the presented tag using ON/OFF coding scheme [16], for LRS/HRS. Fig. 6 shows simulated (full wave) RCS response of proposed chipless tag, for some selected states only, to maintain brevity.

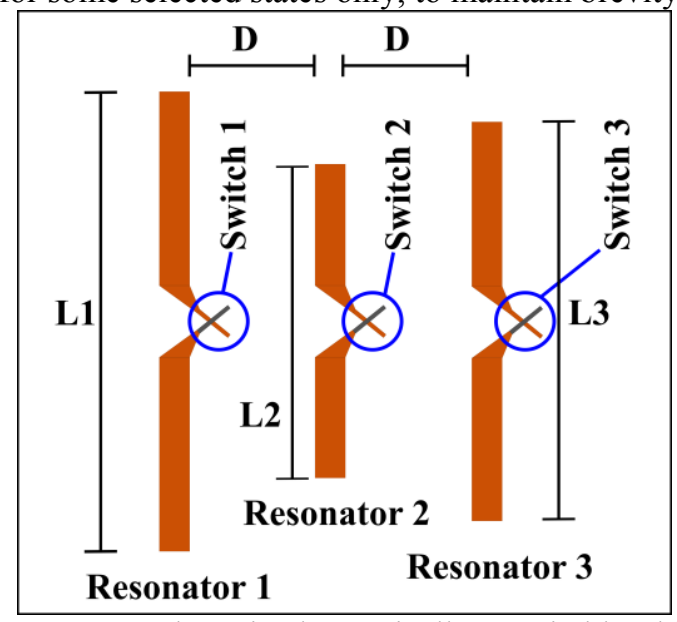

Fig. 5. Proposed 3-Bit electronically rewritable chipless RFID tag using CBRAM/MIM switches. ( $\mathrm{L} 1=40 \mathrm{~mm}$, $\mathrm{L} 2=30 \mathrm{~mm}, \mathrm{~L} 3=35 \mathrm{~mm}, \mathrm{D}=12 \mathrm{~mm}$, refer Fig. 3 for all other dimensions and material details.)

Table 1. Resonance frequencies observed for different switch states for electronically reconfigurable chipless RFID tag presented in Fig. 5.

\begin{tabular}{|c|c|}
\hline $\begin{array}{c}\text { State Number } \\
\text { Resonators switched in the } \\
\text { order of 1, 2, and 3) } \\
0 \rightarrow \text { LRS }(100 \Omega), \\
1 \rightarrow \text { HRS }(1 \mathrm{M} \Omega)\end{array}$ & $\begin{array}{c}\text { Resonance } \\
\text { Frequencies (GHz) }\end{array}$ \\
\hline 000 & No Resonances \\
\hline 001 & 2.944 \\
\hline 010 & 3.256 \\
\hline 011 & $2.944,3.256$ \\
\hline 100 & 2.704 \\
\hline 101 & $2.704,2.944$ \\
\hline 110 & $2.704,3.256$ \\
\hline 111 & $2.704,2.944,3.256$ \\
\hline
\end{tabular}

It is also interesting to note that the calculated ' $Q$ ' factor for each of these resonances are better than 140 and hence this design allows a considerable density of coding per sq.cm of the tag using these rewritable resonators [16]. 


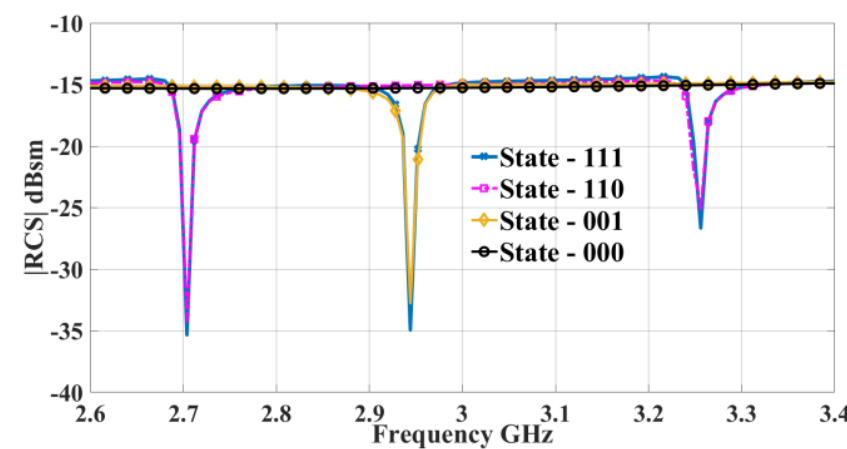

Fig. 6. RCS response of electronically rewritable chipless RFID tag shown in Fig. 5 for different switch states mentioned in Table 1 (full wave simulation).

\section{Conclusion}

In this study we have presented the concept of a pragmatic approach to design of electronically rewritable 'RF-Barcodes' based on non-volatile CBRAM/MIM switches. Presented design corroborates the usage of nonideal ON state impedances of CBRAM/MIM switches for efficient and simple electronically reconfigurable chipless RFID tags. Full wave simulation studies confirm the proposed concept, and experimental realization of presented design with affirmative outcomes is in rapid progress with the authors. We have previously presented the design and realization of RF switches and electronically rewritable RFID tags on rigid as well as flexible substrates in [7], [8]. Previous outcomes from experiments related to reliability of the chosen process [13] confirms positively, exhibiting fully functional 2300 cycles of operation. These promising results and the potential printability of CBRAM/MIM switches along with the chosen strategy, predicts a great future for this technology based new generation of solid state and fully passive RF electronics.

\section{Acknowledgements}

This project has received funding from the European Research Council (ERC) under the European Union's Horizon 2020 research and innovation program (grant agreement No 772539) https://www.scattererid.eu/.

\section{References}

[1] S. Tedjini, N. Karmakar, E. Perret, A. Vena, R. Koswatta, and R. E-Azim, "Hold the Chips: Chipless Technology, an Alternative Technique for RFID," IEEE Microw. Mag., vol. 14, no. 5, pp. 56-65, Jul. 2013, doi: 10.1109/MMM.2013.2259393.

[2] S. Preradovic and N. C. Karmakar, "Chipless RFID: Bar Code of the Future," IEEE Microw. Mag., vol. 11, no. 7, pp. 87-97, Dec. 2010, doi: 10.1109/MMM.2010.938571.

[3] I. Jalaly and I. D. Robertson, "Capacitively-tuned split microstrip resonators for RFID barcodes," in 2005 European Microwave Conference, Paris, France, 2005, vol. 2, doi: 10.1109/EUMC.2005.1610138.

[4] M. Borgese, F. A. Dicandia, F. Costa, S. Genovesi, and G. Manara, "An Inkjet Printed Chipless RFID
Sensor for Wireless Humidity Monitoring," IEEE Sens. J., vol. 17, no. 15, pp. 4699-4707, Aug. 2017, doi: 10.1109/JSEN.2017.2712190.

[5] A. Attaran and R. Rashidzadeh, "Chipless Radio Frequency Identification Tag for IoT Applications," IEEE Internet Things J., vol. 3, no. 6, pp. 13101318, Dec. 2016, doi: 10.1109/JIOT.2016.2589928.

[6] A. Vena, E. Perret, and S. Tedjini, "Chipless RFID Tag Using Hybrid Coding Technique," IEEE Trans. Microw. Theory Tech., vol. 59, no. 12, pp. 33563364, Dec. 2011, doi: 10.1109/TMTT.2011.2171001.

[7] M. P. Jayakrishnan, A. Vena, B. Sorli, and E. Perret, "Solid-State Conductive-Bridging Reconfigurable RF-Encoding Particle for Chipless RFID Applications," IEEE Microw. Wirel. Compon. Lett., vol. 28, no. 6, pp. 506-508, Jun. 2018, doi: 10.1109/LMWC.2018.2830702.

[8] M.P. Jayakrishnan, Arnaud Vena, Brice Sorli, and Etienne Perret, "Electronically Re-Writable Chipless RFID Tag Using Solid State Metal-Insulator-Metal Switches on Paper Substrate," in IEEE/MTT-S International Microwave Symposium (IMS 2019), Boston, M.A., USA, 2019, pp. 400-403, doi: 10.1109/MWSYM.2019.8701004.

[9] S. Yu and H.-S. P. Wong, "Compact Modeling of Conducting-Bridge Random-Access Memory (CBRAM)," IEEE Trans. Electron Devices, vol. 58, no. 5, pp. 1352-1360, May 2011, doi: 10.1109/TED.2011.2116120.

[10] S. Pi, M. Ghadiri-Sadrabadi, J. C. Bardin, and Q. $\mathrm{Xia}$, "Nanoscale memristive radiofrequency switches," Nat. Commun., vol. 6, p. 7519, Jun. 2015, doi: $10.1038 /$ ncomms 8519 .

[11] James Nessel and Richard Lee, "Chalcogenide nanoionic-based radio frequency switch," US 7.923,715 B2, 12-Apr-2011.

[12] L. Chua, "Resistance switching memories are memristors," Appl. Phys. A, vol. 102, no. 4, pp. 765783, Mar. 2011, doi: 10.1007/s00339-011-6264-9.

[13] J. Jian, H. Chang, A. Vena, and B. Sorli, "Study and design of resistive switching behaviors in PMMAbased conducting-bridge random-access memory (CBRAM) devices," Microsyst. Technol., vol. 23, no. 6, pp. 1719-1725, Jun. 2017, doi: 10.1007/s00542015-2754-6.

[14] M. P. Jayakrishnan, A. Vena, A. Meghit, B. Sorli, and E. Perret, "Nafion-Based Fully Passive SolidState Conductive Bridging RF Switch," IEEE Microw. Wirel. Compon. Lett., vol. 27, no. 12, pp. 1104-1106, Dec. 2017, doi: 10.1109/LMWC.2017.2764741.

[15] J. Methapettyparambu Purushothama, "New Generation of Electronically Reconfigurable Passive Microwave Devices Based on Non-volatile CBRAM Switching Technology," PhD Thesis, Université Grenoble Alpes, Valence, France, 2019.

[16] Arnaud Vena, Etienne Perret, and Smail Tedjini, Chipless RFID based on RF Encoding Particle: Realization, Coding and Reading System. New York, NY, USA: ISTE-Elsevier. 